\title{
BMJ Open Exploring the factors that affect new graduates' transition from students to health professionals: a systematic integrative review protocol
}

\author{
Eric Nkansah Opoku (D) , ${ }^{1,2}$ Lana Van Niekerk (D) ,2 \\ Lee-Ann Jacobs-Nzuzi Khuabi (i) ${ }^{2}$
}

To cite: Opoku EN, Van Niekerk L, Jacobs-Nzuzi Khuabi L-A. Exploring the factors that affect new graduates' transition from students to health professionals: a systematic integrative review protocol. BMJ Open 2020;10:e033734. doi:10.1136/ bmjopen-2019-033734

- Prepublication history for this paper is available online. To view these files, please visit the journal online (http://dx.doi. org/10.1136/bmjopen-2019033734).

Received 20 August 2019 Revised 20 March 2020 Accepted 15 May 2020

Check for updates

(c) Author(s) (or their employer(s)) 2020. Re-use permitted under CC BY-NC. No commercial re-use. See rights and permissions. Published by BMJ.

${ }^{1}$ Department of Occupational Therapy, College of Health Sciences; University of Ghana, Accra, Greater Accra Region, Ghana

${ }^{2}$ Faculty of Medicine and Health Sciences, Stellenbosch University, Cape Town, Western Cape, South Africa

Correspondence to Eric Nkansah Opoku; Enopoku@ug.edu.gh

\section{ABSTRACT}

Introduction To become a competent health professional, the nature of new graduates' transition plays a fundamental role. The systematic integrative review will aim to identify the existing literature pertaining to the barriers during transition, the facilitators and the evidencebased coping strategies that assist new graduate health professionals to successfully transition from students to health professionals.

Methods and analysis The integrative review will be conducted using Whittemore and Knafl's integrative review methodology. Boolean search terms have been developed in consultation with an experienced librarian, using Medical Subject Heading terms on Medline. The following electronic databases have been chosen to ensure that all relevant literature are captured for this review: PubMed, EBSCOhost (including Cumulative Index of Nursing and Allied Health Literature (CINAHL), Medline, Academic Search Premier, Health Science: Nursing and Academic Edition), Scopus and Web of Science. A follow-up on the reference list of selected articles will be done to ensure that all relevant literature is included. The Covidence platform will be used to facilitate the process.

Ethics and dissemination Ethical approval is not required for this integrative review since the existing literature will be synthesised. The integrative review will be published in a peer-reviewed journal once all the steps have been completed. The findings will also be presented at international and national conferences to ensure maximum dissemination.

\section{INTRODUCTION}

The transition from undergraduate student to health professional is recognised as a period of great stress for the new graduate. On commencing clinical practice, new graduates enter a relatively new and often challenging environment. They have to make substantial adjustments from being students whose procedures and activities were supervised in a controlled environment to practising independently as qualified health professionals. ${ }^{1}$ This change in status from a student to a health professional is marked
Strengths and limitations of this study

- The search strings for this integrative review were developed in consultation with an experienced librarian.

- The search is comprehensive and will include electronic databases (PubMed, EBSCOhost (including CINAHL, Medline, Health Science: Nursing and Academic Edition), Scopus, Web of Science and Cochrane. Hand searching and follow-up of reference lists of eligible publications will be done to ensure that all relevant literature are included in the study.

- The authors will conduct a blind review to ensure rigorous and consistent application of the inclusion and exclusion criteria.

- Qualitative data analysis software, namely, Weft QDA, will be used to analyse the literature to maximise the findings in terms of both implications for clinical practice and research.

- The findings from the integrative review might not be exhaustive of all available literature on factors that affect new graduates' transition into practice because it will be limited by the inclusion and exclusion criteria.

by changes in both roles and expectations, which requires that the theoretical knowledge acquired in school be transferred to the practice context. ${ }^{2}$ Furthermore, new graduates are expected to plan and implement relevant client treatment programmes. This transition has been described as a period of stress, requiring effective management of conflicting values and role uncertainty. ${ }^{3}$ In a phenomenological study conducted by Brennan et at in the UK, junior doctors who participated in the study described their transition from medical students to junior doctors as extremely stressful; both physically and emotionally. The study highlighted that participants were overwhelmed with feelings of anxiety due to uncertainty about their 
clinical decisions including diagnosis and treatment. ${ }^{4}$ Inadequate preparation for this transition may cause the new health professional's therapy to be ineffective. ${ }^{5}$

The transition to practice for new graduates was described as gradual and complex, involving a complete transformation particularly in the first year after graduation. A thorough literature review by McCombie and Antanavage ${ }^{6}$ established that new graduates experience low personal and professional confidence, particularly at the initial stage of the transition. A phenomenological study by Seah et $a l^{7}$ reported that all participants experienced shock on starting work due to the confusing nature of the hospital facility operations, administrative requirements, expectations of other health professionals and professional title.

To become a competent health professional, the nature of a new graduate's transition plays a fundamental role. Several strategies have been identified in the literature to alleviate the challenges inherent in transition into practice. Supervision has been consistently emphasised as significant during transition. In occupational therapy, supervision has been shown to contribute to new graduates' ability to relate their acquired knowledge to practice. ${ }^{8}$ Hummel and Koelmeyer ${ }^{9}$ conducted a quantitative crosssectional study among occupational therapists $(n=74)$ in Australia to investigate their perceptions, regarding their first year of practice. They found that formal supervision by an experienced health professional, who is capable of providing essential feedback and support, was considered a vital component of a successful transition ${ }^{9}$ and fundamental to the new graduate's perceived success at work. ${ }^{10}$ Tryssenaar and Perkins ${ }^{2}$ also found supervision to be a vital component to promoting competency. Also in the nursing profession, consistent emphasis has been placed on supervision as an effective strategy to help new graduate nurses to relate the knowledge acquired in the classroom to practice. ${ }^{11-13}$ Thus, effective supervision equips new practitioners with competencies that are relevant to their professional career. This impacts on their practice by increasing clinical skills, self-confidence and perception of competence, consequently improving quality of service to clients. ${ }^{12}$

The literature revealed several support and coping strategies aimed at easing the challenges of transition into practice. Moores and Fitzgerald ${ }^{8}$ found that work colleagues play an important role in ensuring successful transition as they provide advice and information to new graduates. Support from experienced colleagues and other new graduate peers was reported to be highly valued. ${ }^{8}$ Halfer and $\mathrm{Graf}^{14}$ confirmed the importance of supervision and emphasised the value of positive relationships with other professionals and coworkers. A thorough literature review by Moores and Fitzgerald ${ }^{8}$ revealed that interactions with peers in the form of group learning, networking and structured discussions on topics relevant to clinical practice supported the transition into practice. In an Australian cross-sectional study by Hummell and Koelmeyer, ${ }^{9}$ the novice occupational therapists $(n=74)$ reported that informal support from other new graduates within and beyond the workplace eased their role transition. Regan $e t a l^{15}$ highlighted that formal orientation and mentorship facilitated new health professionals' transition into practice.

Continued professional development opportunities have also been reported as important in the transition of new graduates into practice. Seah $e t a l^{7}$ reported a positive link between novice professionals' engagement in continued professional development and increased professional confidence in the clinical environment.

An integrative review will be beneficial in examining the availability and the extent of literature, examining varied perspectives on factors that affect transition into practice. It will aim to identify existing literature pertaining to the barriers and facilitators experienced during transition from being a new graduate to becoming a health professional and the evidence-based coping strategies that assist this transition. The integrative review will inform a larger study to be conducted in Ghana on the factors that impact the transition of new health graduates into independent practice. Health professions' education programmes offer different types of graded practical exposure, designed to bridge learning between classroom teaching and clinical practice. For some health professions, clinical practice exposure is embedded in the curricula while others complete the pre-clinical component of their curriculum before entering a distinct internship. These differ in terms of level of expectation, duration and level of independence required. For example, occupational therapists complete 1000 hours of clinical practice experience as part of their education and training. ${ }^{16}$ For the purpose of the integrative review, the authors will consider all practical and clinical exposures required as part of curricula leading up to qualification as entry-level health practitioners to be a component of learning and thus completed in capacity as student health professional. We are interested in the transition health professionals make having completed their education and training into independent practice.

\section{Aims and objectives}

This integrative review aims to identify research conducted in the last two decades (1999-2019) on the factors that affect newly graduated health professionals' transition in becoming health professionals. The specific objectives are:

- To determine the challenges associated with new health graduates' transition into practice.

- To identify the factors that facilitate transition of new health graduates into practice.

- To describe the coping strategies employed by new graduates to ensure successful transition into practice.

\section{METHODS AND ANALYSIS}

The integrative review will be conducted using Whittemore and Knafl's ${ }^{17}$ integrative review methodology as a guide. This methodological framework comprises five 
stages: (1) problem identification, (2) literature search, (3) data evaluation, (4) data analysis and (5) presentation.

\section{Problem identification}

The nature of a health professional's transition into independent practice is critical to development of clinical competence. There is wealth of literature on the transition of new graduates into practice for health professionals from diverse backgrounds. This integrative review will explore the factors that affect newly graduated health professionals' transition into practice comprehensively. To capture the scope and the diversity of available literature,${ }^{17}$ three broad research questions were developed to guide this review. These are:

- What types of challenges do new health graduates face during transition into practice?

- What factors facilitate the transition of new health graduates into practice?

- What coping strategies do new health graduates employ to ensure successful transition into practice?

\section{Literature search}

A search will be done to identify literature from electronic databases. Hand searches will be done to retrieve literature that were not found in the databases. Additionally, a follow-up of the reference lists of the included articles will be done to ensure that all relevant literature are included in the review.

Taking the research question and purpose into consideration, the following electronic databases have been chosen for the search for relevant literature for this review: PubMed, EBSCOhost (including CINAHL, Medline, Academic Search Premier, Health Science: Nursing and Academic Edition), Scopus, Cochrane and Web of Science. These databases and search terms were selected in consultation with an experienced subject librarian. The search terms include Medical Subject Heading terms on Medline (see table 1).

An initial search was done on 3 April 2019 to check the suitability of the search string. These results are presented in table 2. Limiters applied were Published (January 1999 to April 2019), SmartText searching and Language (English only).

The search will be done using the abstract/title field and will include articles published within the last two decades. Hand searching of the reference lists of included

\begin{tabular}{|c|c|}
\hline TOPIC & $\begin{array}{l}\text { 'New graduate' OR ‘Novice Professional' OR } \\
\text { 'Health student' }\end{array}$ \\
\hline AND TOPIC & Transition* \\
\hline AND TOPIC & 'Clinical practice' \\
\hline AND TOPIC & $\begin{array}{l}\text { 'Clinical competence' OR 'Professional } \\
\text { Competence' }\end{array}$ \\
\hline
\end{tabular}

\begin{tabular}{lr}
\hline Table 2 Initial database search results & \\
\hline EBSCOhost & 268 \\
PubMed & 109 \\
Scopus & 66 \\
Web of Science & 68 \\
Cochrane & 2 \\
\hline
\end{tabular}

articles will be done to ensure that all articles relevant to the study are included.

\section{Inclusion criteria}

The review will include only research articles published in English within the last two decades (1999-2019). There will be no restriction by country. Preliminary inclusion and exclusion criteria have been developed as a guide in the selection of studies for this review. These are presented in table 3 .

Only primary studies on transition of new graduate health professionals into practice, when education has been completed, will be included. This is the period between starting work as a supervised novice to being a competent health professional who has completed the transition. Figure 1 illustrates the period of transition into practice.

\section{Data evaluation}

All studies that meet the inclusion criteria will be included in this review. Titles and abstracts of all retrieved literature will be uploaded onto Covidence, which will be used to manage the project. The Covidence platform will remove duplicates automatically before the review process will begin. Quality assessment will consider issues such as the clarity of the study aim, the participants and the relevance to answer the research questions of the proposed review. Three independent reviewers will screen all studies against the inclusion criteria to determine their eligibility to be included in this integrative review. Following the title and abstract screening, the full texts of the included publications will be uploaded for full-text screening against the same predetermined inclusion and exclusion criteria by

\section{Table 3 Provisional selection criteria}

\begin{tabular}{ll}
\hline Inclusion criteria & Exclusion criteria \\
\hline $\begin{array}{l}\text { Peer-reviewed research articles } \\
\text { on new health graduates' } \\
\text { transition from novice to } \\
\text { experienced health professionals. }\end{array}$ & $\begin{array}{l}\text { Conference abstracts, } \\
\text { doctoral theses and grey } \\
\text { literature on new health } \\
\text { graduates' transition to } \\
\text { practice. }\end{array}$ \\
$\begin{array}{ll}\text { Primary sources of systematic } \\
\text { reviews that meet the inclusion } \\
\text { criteria will be included. }\end{array}$ & $\begin{array}{l}\text { Systematic or literature } \\
\text { reviews on new health } \\
\text { graduates' transition to } \\
\text { practice. }\end{array}$
\end{tabular}

Peer-reviewed articles published

in English in the last two

decades. 


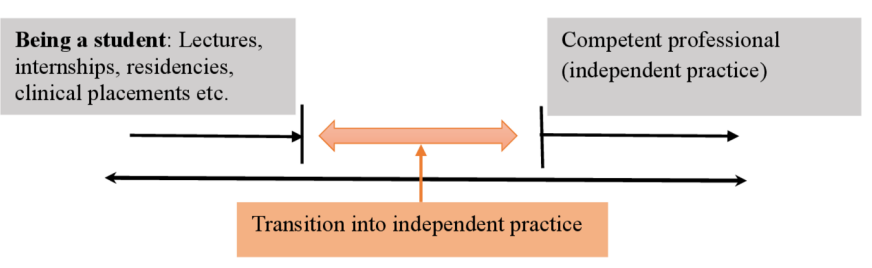

Figure 1 Period of transition into practice illustration.

the same three reviewers. Full-text publications which meet the inclusion criteria will be selected for data extraction. Conflicts will be resolved by consultation among the three reviewers until a consensus is reached. The Preferred Reporting Items for Systematic Reviews and Meta-Analysis Protocols diagram will be used to represent the number of articles that were identified in each of the steps of the review process for visual representation.

Once consensus has been reached on eligibility, relevant data will be extracted. The primary reviewer will read each of the selected full-text publications. A data charting form, adapted from Uys $e t a l^{18}$ will be used in the extraction of the data that will answer the research questions and organise existing literature. To test the feasibility, the form will be tested by the lead reviewer on a random sample of the publications included in the review. Key information obtained from the full articles reviewed will be charted. For each of the included studies, the researcher will extract the following data:

1. Study characteristics: author names, publishing journal, year of study published, country of study and the population of the study.

2. Study aims, objectives and/or research questions, the study design.

3. The findings, with particular emphasis on the barriers, the facilitators and coping strategies of transition into practice.

\section{Data analysis}

An inductive and thematic approach to data analysis will be used. Weft QDA, a qualitative data analysis system, will be used to analyse and categorise the literature into theme areas. The analysis will focus on extracting themes from the following areas:

- The challenges associated with transition into practice for new health graduates.

- The facilitators of transition into practice for new health graduates.

- The coping strategies employed by new graduates to ensure successful transition into practice.

\section{Presentation}

Once the data evaluation and extraction process have been completed, the study findings will be presented in the form of descriptions and narrations. Given the likely heterogeneity of studies that will be included in this study, narrative summaries of the characteristics of each study will be presented. The findings will be synthesised and written up into a coherent article.

\section{Patient and public involvement}

The proposed integrative review will not have any patient or public involvement.

\section{DISCUSSION}

The researchers anticipate that the findings of this integrative review will contribute to advancing knowledge of the barriers, the facilitators and the coping strategies of transition into practice. These can enable clinical supervisors, academics and policymakers to better understand challenges faced and strategies that can assist novice health professionals. Students and new graduates in health sciences might be informed of the obstacles that they are likely to encounter when they commence practice after graduation. They might also be informed of the facilitators and coping strategies that have been found to assist transition into practice. Findings from this study might also inform curriculum development to better prepare students for transition into practice.

\section{Ethics and dissemination}

Ethical approval is not required for this integrative review since existing literature will be synthesised. Once all the steps have been completed, the integrative review will be published in health professional education or practice journal. The findings will also be presented at international, national and local conferences.

Twitter Eric Nkansah Opoku @0pokuNkansah

Acknowledgements The authors acknowledge the assistance of Mrs Ingrid Van der Westhulzen (subject librarian) at the University of Stellenbosch for contributing towards developing the initial search strings for the integrative review.

Contributors All three authors of this article contributed to the conceptualisation, drafting, development and editing of this integrative review protocol. ENO drafted the initial protocol manuscript as part of his master's degree, LVN and L-AJ-NK guided the development of the protocol and made substantial conceptual and editing contributions and have approved this manuscript. All researchers contributed to all drafts of the manuscript and will be involved in screening and extracting the data once the integrative review commences. The researchers are all committed to being accountable for all aspects of this protocol.

Funding The authors have not declared a specific grant for this research from any funding agency in the public, commercial or not-for-profit sectors.

Competing interests None declared.

Patient and public involvement Patients and/or the public were not involved in the design, or conduct, or reporting, or dissemination plans of this research.

Patient consent for publication Not required.

Provenance and peer review Not commissioned; externally peer reviewed.

Open access This is an open access article distributed in accordance with the Creative Commons Attribution Non Commercial (CC BY-NC 4.0) license, which permits others to distribute, remix, adapt, build upon this work non-commercially, and license their derivative works on different terms, provided the original work is properly cited, appropriate credit is given, any changes made indicated, and the use is non-commercial. See: http://creativecommons.org/licenses/by-nc/4.0/.

\section{ORCID iDs}

Eric Nkansah Opoku http://orcid.org/0000-0002-8970-0059

Lana Van Niekerk http://orcid.org/orcid.org/0000-0003-0003-6006

Lee-Ann Jacobs-Nzuzi Khuabi http://orcid.org/0000-0003-0684-5373 


\section{REFERENCES}

1 Naidoo D, Van WJ, Joubert RN. Are final year occupational therapy students adequately prepared for clinical practice? A case study in KwaZulu-Natal. South African J Occup Ther 2014;44.

2 Tryssenaar J, Perkins J. From student to therapist: exploring the first year of practice. Am J Occup Ther 2001;55:19-27.

3 Morley M, Rugg S, Drew J. Before Preceptorship: new occupational therapists' expectations of practice and experience of supervision. Br J Occup Ther 2007;70:243-53.

4 Brennan N, Corrigan O, Allard J, et al. The transition from medical student to junior doctor: today's experiences of tomorrow's doctors. Med Educ 2010;44:449-58.

5 Naidoo D, Van Wyk J. Fieldwork practice for learning: lessons from occupational therapy students and their supervisors. AJHPE 2016;8:37.

6 McCombie RP, Antanavage ME. Transitioning from occupational therapy student to practicing occupational therapist: first year of employment. Occup Ther Health Care 2017;31:126-42 https://doi. org/

7 Seah $\mathrm{CH}$, Mackenzie L, Gamble J. Transition of graduates of the master of occupational therapy to practice. Aust Occup Ther $J$ 2011:58:103-10.

8 Moores A, Fitzgerald C. New graduate transition to practice: how can the literature inform support strategies? Aust Heal Rev 2017;41:308-12
9 Hummell J, Koelmeyer L. New graduates: perceptions of their first occupational therapy position. Br J Occup Ther 1999;62:351-8.

10 Hunt K, Kennedy-Jones M. Novice occupational therapists perceptions of readiness to undertake fieldwork supervision. Aust Occup Ther J 2010;57:394-400.

11 De Bellis A, Longson D, Glover P, et al. The enculturation of our nursing graduates. Contemp Nurse 2001;11:84-94.

12 Melman S, Ashby SE, James C. Supervision in practice education and transition to practice: student and new graduate perceptions. Internet J Allied Heal Sci Pract 2016;14.

13 Al Awaisi H, Cooke H, Pryjmachuk S. The experiences of newly graduated nurses during their first year of practice in the Sultanate of Oman - A case study. Int J Nurs Stud 2015;52:1723-34.

14 Halfer D, Graf E. Graduate nurse perceptions of the work experience. Nurs Econ 2006;24:150-5.

15 Regan S, Wong C, Laschinger HK, et al. Starting out: qualitative perspectives of new graduate nurses and nurse leaders on transition to practice. J Nurs Manag 2017;25:246-55.

16 Minimum Standards for the Education of Occupational Therapists. Council of the world Federation of occupational therapists. world Federation of occupational therapists 2016.

17 Whittemore R, Knafl K. The integrative review : updated methodology. Methodol issues Nurs Res 2005;52:546-53.

18 Uys ME, Buchanan H, Van Niekerk L. Strategies occupational therapists employ to facilitate work-related transitions for persons with hand injuries: a study protocol for a scoping review. BMJ Open 2019;9:2-7 